\title{
Tumor-associated macrophages in clear cell renal cell carcinoma express both gastrin-releasing peptide and its receptor: a possible modulatory role of immune effectors cells
}

\author{
Jens Bedke • Bernhard Hemmerlein · Christina Perske • \\ Andreas Gross · Markus Heuser
}

Received: 4 September 2008 / Accepted: 16 November 2009 / Published online: 13 December 2009

(C) The Author(s) 2009. This article is published with open access at Springerlink.com

\begin{abstract}
Purpose Renal cell carcinomas (RCC) frequently express the gastrin-releasing peptide receptor (GRP-R). Gastrinreleasing peptide (GRP) stimulates tumor cell proliferation and neoangiogenesis. Tumor-associated macrophages (TAM) comprise an important cellular component of these tumors. We analyzed the GRP/GRP-R network in clear cell RCC (ccRCC) and non-clear cell RCC (non-ccRCC) with special regard to its expression by macrophages, tumor cells and microvessels.

Methods Gastrin-releasing peptide and GRP-R expression in 17 ccRCC and 9 non-ccRCC were analyzed by RT-PCR, immunohistochemistry and double immunofluorescence staining.

Results Tumor-associated macrophages expressed GRP and GRP receptor in ccRCC. Tumor cells and microvessels showed low to intermediate GRP-R expression in nearly all cases. In 12 ccRCC tumor epithelia also expressed low
\end{abstract}

J. Bedke and B. Hemmerlein contributed equally to this work.

J. Bedke

Department of Urology,

Eberhard Karls University of Tübingen,

Tübingen, Germany

B. Hemmerlein · C. Perske

Department of Pathology,

Georg-August-University of Göttingen,

Göttingen, Germany

A. Gross

Department of Urology, Asklepios Hospital Barmbek,

Hamburg, Germany

\section{Heuser ( $\square)$}

Department of Urology, Städtisches Klinikum Solingen, Gotenstr. 1, 42653 Solingen, Germany

e-mail: urologie@klinikumsolingen.de; mheuser@gwdg.de levels of GRP. Microvascular GRP expression was found in nine cases of ccRCC. For non-RCC, the expression of GRP and GRP receptor expression pattern was similar.

Conclusions Tumor-associated macrophages are the main source of GRP in RCC. GRP receptor on TAM, tumor epithelia and microvessels might be a molecular base of a GRP/GRP receptor network, potentially acting as a paracrine/autocrine modulator of TAM recruitment, tumor growth and neoangiogenesis.

Keywords Tumor-associated macrophages .

Renal cell carcinoma Gastrin-releasing peptide .

Growth factor

\section{Introduction}

Gastrin-releasing peptide (GRP) is the human analog of the amphibian tetradecapeptide bombesin. McDonald et al. [1] was the first to describe GRP in the gastrointestinal tract of mammals. GRP, originally named for its ability to stimulate the release of gastrin, modulates the secretion of a variety of gastrointestinal hormones and affects pancreatic secretion, intestinal transit, muscle contractility, metabolism and behavior [2]. The expression of GRP is not only restricted to the gastrointestinal tract and it is also widely distributed in the central nervous system.

GRP interacts with four different G-protein coupled receptors: the GRP receptor (subtype I), the neuromedin-B receptor, the Bombesin receptor (subtype 3 ), and a novel subtype 4 that has been identified in frogs. In humans, GRP binds to the GRP receptor with high affinity and to the subtype 3 receptor with low affinity.

GRP has been identified as an important growth factor not only for neuroendocrine malignancies, such as medullary 
thyroid carcinomas [3], small cell lung carcinomas [4] and neuroblastomas [5], but also for carcinomas of the breast [6], colon [7], prostate [8] and rat pancreatic cancer [9]. The growth of renal cell carcinoma (RCC) has been linked to the presence of GRP and its receptor by Pansky et al. [10] who stimulated the growth of various RCC cell lines with GRP. Reubi et al. [11] identified the GRP receptor on tumor epithelia in human RCC specimens using a radioactive ligand. We have shown recently that the GRP receptor is not only found on tumor epithelia, but also expressed in microvessels [12]. We likewise demonstrated that the microvascular GRP receptor is potentially relevant for neoangiogenesis in RCC, because microvessel formation is inhibited by the GRP receptor antagonist RC-3095 in an animal model of a dorsal skinfold chamber.

Malignant tumors comprise up to 50\% stromal cells of which monocytes/macrophages are a quantitative and functional important subpopulation. The tumor microenvironment and hypoxia induce a differentiation of tumorassociated macrophages (TAM) into a phenotype with depressed cytotoxicity [13-15] which may enhance growth and spread of tumor cells [16-18].

Neuropeptides, e.g. GRP, significantly modulate the function of macrophages. The GRP receptor has been found on macrophages, and GRP has an effect on macrophage function such as displayed by an alteration of adhesion, migration and phagocytosis [19, 20]. Interestingly, GRP expression in macrophages has been reported only under inflammatory conditions, such as chronic bronchitis and lung inflammation and fibrosis [21, 22]. In inflammation, the findings of simultaneous peptide and receptor expression suggest an autocrine loop in the macrophage environment that may be important for the macrophage/epithelium interaction.

In the present study, we confirm the presence of GRP and GRP receptor mRNA transcripts in tumor tissue of human clear cell RCC (ccRCC) and non-clear cell RCC (non-ccRCC). Moreover, as novel finding, we co-localize an expression of GRP and GRP-R in TAM and also in microvessels. We conclude that the GRP/GRP receptor system in RCC and TAM may represent a link between growth regulation in the tumor and local immune response.

\section{Materials and methods}

Patients and tumors

Seventeen ccRCC and nine non-ccRCC were freshly obtained at the time of surgery from the urological department of our institution. Approval of the ethics committee had been obtained before initiation of the study. Histopathological data are summarized in Table 1.
Immunohistochemistry and immunofluorescence

Tissues were fixed in HOPETM (Hepes-glutamic acid buffer-mediated organic solvent protection effect) solution (DCS Diagnostic Systems, Hamburg, Germany) and embedded in paraffin according to the manufacturer's instructions. Tissue sections $(3 \mu \mathrm{m})$ were mounted on glass slides, de-paraffinized, rehydrated and subjected to immunohistochemistry. Sections were incubated with a polyclonal rabbit anti-gastrin-releasing peptide antibody (1:50 dilution, Dako, Hamburg Germany) or polyclonal rabbit anti-gastrin-releasing peptide receptor (GRP-R) antibody (1:200 dilution; kindly donated by Dr. Battey, NIDCD, Rockville, MD) at room temperature for $2 \mathrm{~h}$. After several washing steps, the sections were subsequently incubated with Envision ${ }^{\mathrm{TM}}$ peroxidase (Dako) for $30 \mathrm{~min}$. Signals were detected with diaminobenzidine as substrate (Dako). Finally, sections were counterstained with hematoxylin, dehydrated with ethanol and xylene and covered with cover slips.

Immunohistochemical staining for GRP and its receptor was semiquantitatively analyzed by means of an immunoreaction score: 0 , no staining; 1 staining $<10 \%$ of cells; 2 $10-50 \%$ of cells stained; $3>50 \%$ of cells stained.

For double immunofluorescence analysis, sections were incubated with a mixture of anti-GRP (1:25) and CD68 (1:3,000, Dako Clone KP-1) at $4^{\circ} \mathrm{C}$ overnight. Anti-CD 68 antibody was detected with an anti-mouse $\mathrm{Cy} 3$ conjugate (1:500 dilution for $1 \mathrm{~h}$ at RT). Subsequently, the sections were incubated with anti-rabbit Envision-Peroxidase (Dako) for $1 \mathrm{~h}$ at RT and incubated with tyramide-FITC to detect GRP (tyramid signal amplification, PerkinElmer/ NEN, Rodgau, Germany). Finally, sections were mounted with fluoromount (Dako). Immunofluorescence stainings were visualized by means of an Axiophot fluorescence microscope (Zeiss, Göttingen, Germany) and a fluorescence camera (Fview, Olympus, Hamburg, Germany).

mRNA expression analysis

Paraffin sections of HOPE-fixed tissue were cut under RNAse-free conditions and transferred to reaction tubes. The sections were de-paraffinized with three changes of isopropanol at $60^{\circ} \mathrm{C}$ for at least $90 \mathrm{~min}$. The sections were washed with absolute ethanol, air dried and lysed in lysis buffer applying the RNAeasy mini kit (Qiagen, Hilden, Germany), and RNA was extracted according to the manufacturer's instructions. The quality and quantity of isolated RNA were tested in an automated capillary electrophoresis system (Agilent BioAnalyzer 2100; Agilent Technologies, Waldbronn, Germany). A maximum of $500 \mathrm{ng}$ of total RNA was reverse transcribed using random hexamer primers (Invitrogen, Karlsruhe, Germany) and Omniscript ${ }^{\mathrm{TM}}$ 
Table 1 Synopsis of clinicopathological data and expression analysis of ccRCC and non-ccRCC

\begin{tabular}{|c|c|c|c|c|c|c|c|c|c|c|c|c|}
\hline \multirow[t]{3}{*}{ CcRCC } & \multirow[t]{3}{*}{ Grade } & \multirow[t]{3}{*}{ pTNM } & \multirow{2}{*}{\multicolumn{2}{|c|}{ Tumor cells }} & \multicolumn{5}{|c|}{ Stromal cells } & \multirow{2}{*}{\multicolumn{3}{|c|}{ RNA }} \\
\hline & & & & & \multicolumn{3}{|c|}{ Macrophages } & \multicolumn{2}{|c|}{ Vessels } & & & \\
\hline & & & GRP & GRP-R & GRP & \multicolumn{2}{|c|}{ GRP-R } & GRP & GRP-R & GRP & GRP-R & PBGD \\
\hline \multicolumn{13}{|l|}{ Case } \\
\hline 1 & G1 & pT1b, Nx, Mx & 1 & 1 & 1 & \multicolumn{2}{|c|}{ n.a. } & 0 & 3 & 2 & 2 & 2 \\
\hline 2 & G1 & pT1a, Nx, Mx & 1 & 2 & 0 & \multicolumn{2}{|c|}{1} & 1 & 1 & 0 & 0 & 3 \\
\hline 3 & G1 & pT1b, Nx, Mx & 0 & 2 & 3 & 2 & & 1 & 2 & 2 & 3 & 2 \\
\hline 4 & G2 & pT2, Nx, M1 & 0 & 2 & 3 & 3 & & 1 & 2 & 3 & 3 & 3 \\
\hline 5 & $\mathrm{G} 2$ & pT3a, N0, Mx & 1 & 1 & 1 & 0 & & 1 & 2 & 2 & 2 & 2 \\
\hline 6 & G2 & $\mathrm{pT} 2, \mathrm{Nx}, \mathrm{M} 1$ & 1 & 1 & 2 & 3 & & 0 & 1 & 3 & 3 & 2 \\
\hline 7 & $\mathrm{G} 2$ & pT1b, Nx, Mx & 1 & 1 & 1 & 1 & & 0 & 2 & 2 & 2 & 2 \\
\hline 8 & G2 & $\mathrm{pT} 2, \mathrm{Nx}, \mathrm{Mx}$ & 0 & 1 & 3 & 2 & & 0 & 1 & 2 & 2 & 2 \\
\hline 9 & G2 & pT3a, Nx, Mx & 1 & 2 & 1 & 2 & & 1 & 2 & 2 & 3 & 2 \\
\hline 10 & $\mathrm{G} 2$ & pT3b, Nx, Mx & 1 & 1 & 1. & 2 & & 1 & 1 & 2 & 2 & 2 \\
\hline 11 & $\mathrm{G} 2-3$ & pT3b, Nx, Mx & 1 & 1 & 0 & 2 & & 0 & 1 & Degrad & RNA & \\
\hline 12 & G2-3 & pT3b, Nx, Mx & 1 & 1 & 2 & 2 & & 0 & 2 & 2 & 2 & 2 \\
\hline 13 & $\mathrm{G} 2-3$ & $\mathrm{pT} 2, \mathrm{Nx}, \mathrm{Mx}$ & 0 & 2 & 2 & 2 & & 0 & 1 & 3 & 3 & 2 \\
\hline 14 & G3 & pT3b, Nx, M1 & 1 & 1 & 2 & 2 & & 2 & 1 & 3 & 3 & 2 \\
\hline 15 & G3 & pT3b, Nx, Mx & 1 & 1 & 3 & 3 & & 1 & 2 & 2 & 3 & 2 \\
\hline 16 & G2 & pT1b, Nx, Mx & 0 & 2 & 2 & 2 & & 1 & 2 & 2 & 3 & 2 \\
\hline 17 & & pT3b, Nx, M1 & 1 & 1 & 1 & 2 & & 0 & 1 & 3 & 2 & 3 \\
\hline Non-ccRCC & Tumor & pTNM & Tumo & r cells & & Strom & cells & & & RNA & & \\
\hline & & & & & & Macr & nages & & & & & \\
\hline & & & GRP & GRP C & & GRP & GRP-R & & GRP-R & GRP & GRP-R & PBGD \\
\hline Case & & & & & & & & & & & & \\
\hline 18 & pap & $\mathrm{pT} 2, \mathrm{Nx}, \mathrm{Mx}$ & 2 & 1 & & 0 & 1 & 0 & 1 & 3 & 2 & 3 \\
\hline 19 & $\mathrm{chr}$ & $\mathrm{pT} 3 \mathrm{~b}, \mathrm{~N} 2, \mathrm{M} 1$ & 0 & 2 & & 3 & 3 & 2 & 3 & 2 & 3 & 2 \\
\hline 20 & sarc & pT4, Nx, Mx & 1 & 1 & & 2 & 3 & 0 & 1 & 2 & 2 & 2 \\
\hline 21 & sarc & pT3a, N2, Mx & 1 & 2 & & 3 & 3 & 0 & 2 & 2 & 2 & 3 \\
\hline 22 & sarc & $\mathrm{pT} 2, \mathrm{~N} 0, \mathrm{Mx}$ & 0 & 3 & & 2 & 2 & 0 & 1 & 3 & 3 & 2 \\
\hline 23 & sarc & pT3a, N2, M1 & 0 & 1 & & 3 & 3 & 0 & 1 & 3 & 2 & 3 \\
\hline 24 & sarc & pT3a, Nx, M1 & 2 & 2 & & 2 & 3 & 0 & 1 & 0 & 1 & 1 \\
\hline 25 & $\mathrm{db}$ & $\mathrm{pT} 3 \mathrm{~b}, \mathrm{~N} 2, \mathrm{Mx}$ & 1 & 3 & & 3 & 2 & 0 & 2 & 0 & 2 & 3 \\
\hline 26 & $\mathrm{db}$ & pT3a, Nx, Mx & 0 & 2 & & 1 & 2 & 2 & 2 & 2 & 3 & 2 \\
\hline
\end{tabular}

pap papillary carcinoma, sarc sarcomatoid carcinoma. $d b$ Duct Bellini carcinoma, n.a. not applicable

reverse transcriptase (Qiagen) in a $20 \mu \mathrm{l}$ volume ( $25 \mathrm{ng}$ $\mathrm{RNA} / \mu \mathrm{l})$ at $37^{\circ} \mathrm{C}$ for $60 \mathrm{~min}$ according to the manufacturer's instructions.

A $25 \mathrm{ng}$ of cDNA was subjected to PCR analysis in a total volume of $20 \mu \mathrm{l}$ using Hotstart Mastermix (Qiagen) and primer pairs with conditions as summarized in Table 2. Porphobilinogen deaminase was used as a house-keeping gene which is constantly expressed in renal carcinomas. PCR products were separated on a $1.5 \%$ agarose gel, stained with ethidium bromide and analyzed on a UV transilluminator. The used primers are given in Table 2.

\section{Statistics}

For the analysis of the immunohistological staining, the oneway ANOVA with Dunnett's post test was performed using GraphPad Prism version 4.00 for Windows, GraphPad Software, San Diego CA USA, http://www.graphpad.com. 
Table 2 Used primers in PCR

\begin{tabular}{clll}
\hline Gene & Sequence $\left(5^{\prime}-3^{\prime}\right)$ & $T_{\mathrm{m}}\left({ }^{\circ} \mathrm{C}\right)$ & $\begin{array}{l}\text { Product } \\
\text { length }(\mathrm{bp})\end{array}$ \\
\hline GRP & & 64 & 286 \\
fW & tgggcggtggggcactta & & \\
rv & tcagctgggggttccttcct & & \\
GRP-R & & 61 & 389 \\
fw & ctcccegtgaacgatgactgg & & \\
rv & atcttcatcagggcatgggag & & 127 \\
PBGD & & 72 & \\
fw & tgtctggtaacggcaatgcggctgcaac & 72 \\
rv & tcaatgttgccaccacactgtccgtct & & \\
\hline
\end{tabular}

\section{Results}

mRNA expression analysis of GRP and GRP receptor in ccRCC

mRNA expression analysis of GRP and GRP receptor was performed in serial sections of the same tissue blocks used for immunohistochemistry. Particularly for GRP, we could demonstrate that GRP is synthesised within the tumor environment of ccRCC and non-ccRCC (Fig. 1; Table 1).

Immunohistochemical localization of GRP and GRP receptor in ccRCC

In ccRCC tumor cells with a low GRP signal were observed in 12 cases. The mean GRP scores in tumor cells (score ${ }^{\text {mean }}$ 0.71 ) and tumor vessels (score ${ }^{\text {mean }} 0.59$ ) were significantly lower than in TAM (score $\left.{ }^{\text {mean }} 1.65\right)(P<0.01)$. Therefore, TAM were the main source of GRP in ccRCC.

Addressing the question which cell types express GRP receptor, a low to high signal intensity was detected in
ccRCC. The mean GRP receptor expression was significantly lower in tumor epithelia (score ${ }^{\text {mean }} 1.35$ ) than in TAM (score $\left.{ }^{\text {mean }} 1.94\right)(P<0.0 .5)$ and lower in association with tumor microvessels (score ${ }^{\text {mean }} 1.58$ ).

In non-ccRCC, a low GRP signal of tumor cells was detected in three cases and an intermediate signal in two cases. The mean GRP scores in non-ccRCC tumor cells (score $\left.^{\text {mean }} 0.78\right)$ and tumor vessels $\left(\right.$ score $\left.^{\text {mean }} 0.44\right)$ were significantly lower than in TAM (score $\left.{ }^{\text {mean }} 2.11\right)(P<0.05)$. For GRP-R expression, non-ccRCC exhibited the same pattern as ccRCC, but no significant difference was detected (data not shown).

When comparing immunohistochemically stained serial sections, we also found GRP and GRP receptor-positive macrophages and GRP receptor-positive tumor cells located in close vicinity (Fig. 2).

To confirm this, we performed double immunofluorescence staining with CD 68 and GRP antibodies macrophages within RCC expressed both proteins on their surface as demonstrated by the orange composite signaling in confocal microscopy (Fig. 2).

Comparative analyses of the degree of differentiation, and the tumor stage did not reveal any correlation. The data on all patients are summarized in Table 1.

Analysis of tumor-free tissues of nine cases revealed a strong GRP and GRP-R expression in tubules and collecting ducts in contrast to peritubular capillaries where no expression could be found. No GRP or GRP receptor-positive macrophages were found in tumor-free tissue.

\section{Discussion}

The present study provides evidence that GRP and its highaffinity receptor are simultaneously expressed in renal carcinomas. The staining pattern of tumor cells and microvascular endothelia confirmed our previous results on the
Fig. 1 Gel electrophoresis of RT-PCR products of typical cases separated on a $1.5 \%$ agarose gel. Blots have been resorted into groups of ccRCC and non-ccRCC. A summary of the cases is shown in Table 1

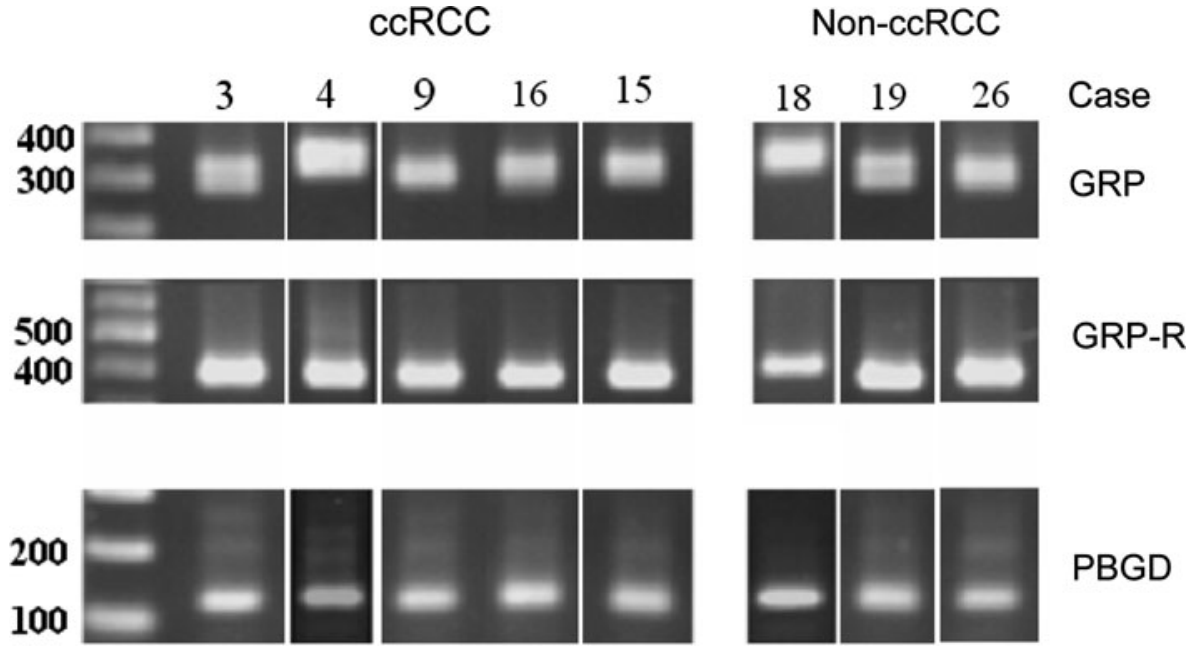


Fig. 2 Immunohistochemistry of GRP and GRP receptor of case 15 (ccRCC a, b),

19 (chromophobe RCC c, d) and case 18 (papillary RCC e, f) $(\times 400)$. GRP expression is shown in a, $\mathbf{c}$, and e. GRP-R expression is demonstrated in $\mathbf{b}$, d, and f. In case 15 (ccRCC), GRP and GRP-R are expressed by tumor cells, microvessels (right arrow), and with highest intensity by interstitial macrophages (filled triangle) which is highlighted in the figure inlets (a, b). In case 19 (chromophobe RCC) GRP is expressed by foamy (asterisk) and perivascular (filled triangle) macrophages, microvessels (right arrow), but not by tumor cells. Endothelial GRP expression is highlighted in the figure inlet (a). In case 19, GRP-R is expressed by foamy (asterisk) and perivascular (filled triangle) macrophages, microvessels (right arrow), and with faint signals by tumor cells (b). In case 18, GRP is expressed by tumor cells, but not by foamy macrophages $(X)(\mathbf{c})$. GRP-R signals are only faintly seen in macrophages $(X)$ and tumor cells (d). For detailed description see Table 1. g-i Double immunofluorescence staining of GRP and CD68 in a papillary renal carcinoma, case $18(\times 400)$. CD68-positive interstitial macrophages are shown in g (red fluorescence). GRP signals are demonstrated in h (green fluorescence). Overlay of $\mathbf{g}$ and $\mathbf{h}$ confines that interstitial macrophages express GRP (i)
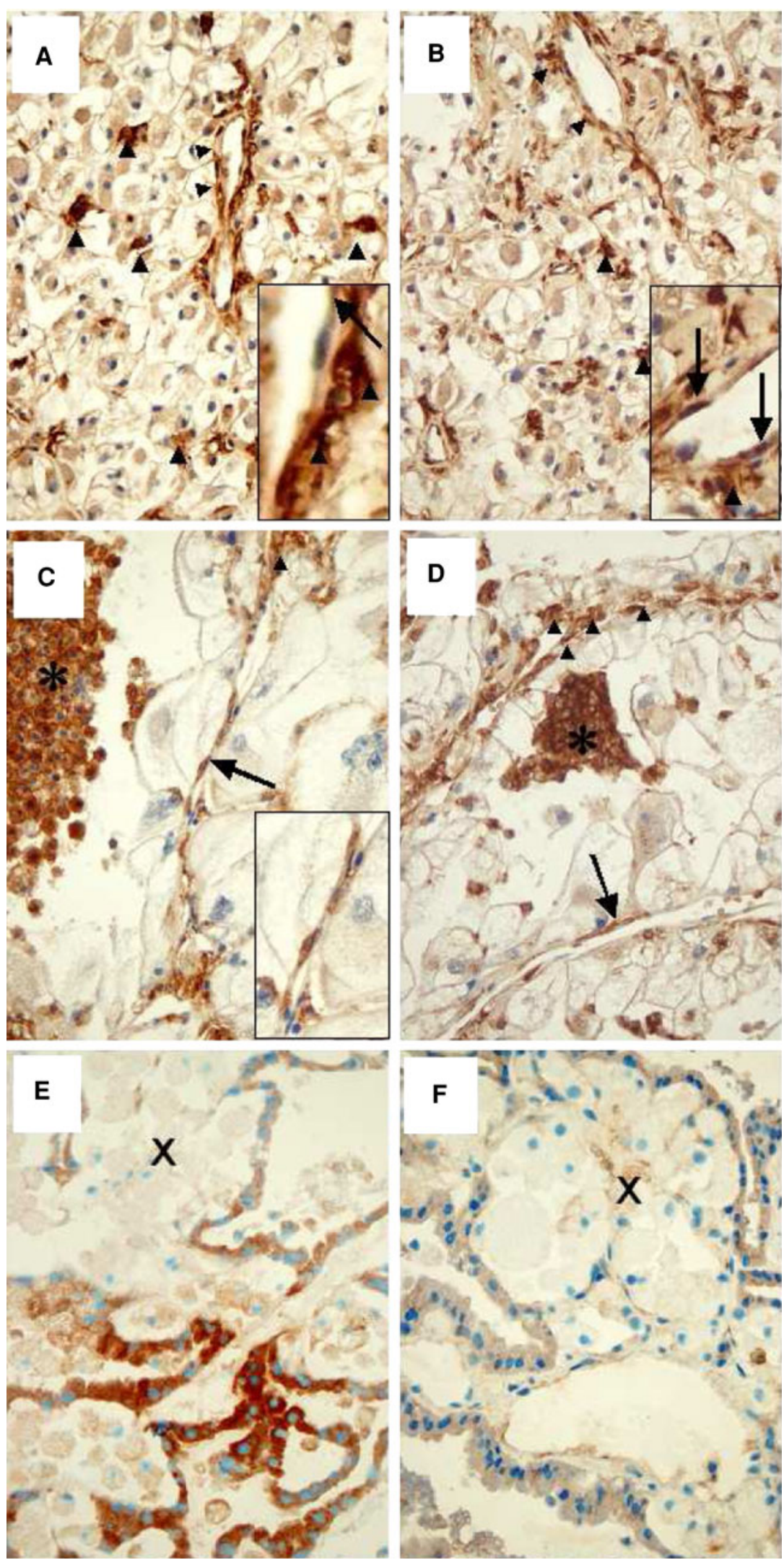
Fig. 2 continued
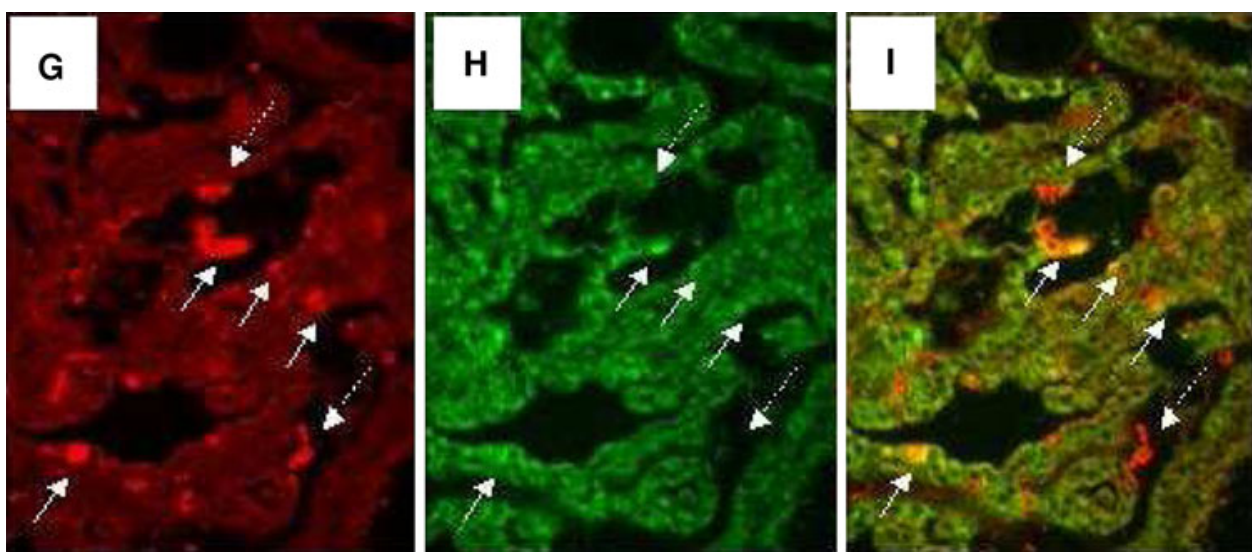

GRP receptor expression in 18 ccRCC [12]. In the present study, we used HOPE fixation, which allowed the simultaneous detection of specific mRNA transcripts and of proteins by means of RT-PCR and immunohistochemistry in 17 ccRCC and 9 non-ccRCC.

In most cases, we found a co-expression of mRNA and protein. Only in cases with RNA degradation and hypoxic necrosis differences between mRNA and protein expression were observed. Protein expression was located nearby or within the necrotic rims. The absence of mRNA expression for GRP can be explained by an advanced degradation of mRNA, even in cases with detectable PBGD mRNA which is abundantly expressed in renal carcinomas.

Immunohistochemical analysis of the GRP receptor expression has been described previously. Detection of the ligand using a polyclonal GRP antiserum has also been reported [23, 24]. We found cytoplasmatic signals for GRP, particularly in TAM and in lower intensity in tumor epithelia. Endothelial expression was found at low levels in few cases, predominately in ccRCC. GRP receptor expression analysis was positive, particularly in TAM and microvessels and with lower intensity in tumor cells of all cases. The discontinuous and granular staining for the GRP receptor was in contrast to the study of Scott et al. [25] who found a more homogeneous cytoplasmatic staining for the GRP receptor in carcinoid tumor epithelia, whereas the staining pattern for GRP corresponded well to our data. GRP and GRP expression has been analyzed in a broad number of neoplasias.

Up til now, investigators did not pay attention on the expression of GRP and GRP-R on macrophages and microvessels. The expression of bombesin/GRP in immune cells has been identified in circulating peripheral monocytes and in alveolar macrophages [26]. Furthermore, GRP acts as chemoattractant for murine peritoneal macrophages [20] and potentially stimulates cytotoxic natural killer cells both in vivo and in vitro [27]. In contrast, Meloni et al. [26] showed that bombesin/GRP stimulates the release of interleukin-8 (IL-8) by peripheral monocytes and may thereby promote neoangiogenesis and tumor growth indirectly. In renal carcinomas, a high number of infiltrating monocytes and macrophages have already been identified [28]. The stimulation of the GRP-R induced the release of interleukin- 8 and vascular endothelial growth factor in human prostate cancer cell lines [29].

The detection of the GRP-R on tumor microvessels underlines the importance of GRP for the progression of renal carcinomas and suggests the presence of an autocrine or paracrine loop within the tumor. We have shown previously that the density of perfused microvessels and tumor blood flow are reduced if a specific GRP receptor antagonist is administered into nude mice bearing subcutaneously implanted renal carcinomas [12]. However, our finding has been questioned by Busby et al. [30] who demonstrated that bombesin/GRP did not have any mitogenic effect on isolated human vascular endothelial cells (HUVEC). This discrepancy may be explained by the fact that HUVEC originate from the umbilical cord and the findings that we have also identified GRP receptors on tumor pericytes that may also be involved in the proliferation of endothelia.

In conclusion, our study confirms the presence of both GRP and GRP receptor in ccRCC. TAM are the main source of intratumoral GRP. The results suggest a paracrine/autocrine signaling within renal carcinomas between TAM, tumor epithelia and microvessels; whereas, the functional relevance of GRP receptors in microvessels and tumor epithelia has been investigated, the significance and the function of the GRP receptor expression in macrophages needs further elucidation.

\section{Conflict of interest statement None.}

Open Access This article is distributed under the terms of the Creative Commons Attribution Noncommercial License which permits any noncommercial use, distribution, and reproduction in any medium, provided the original author(s) and source are credited.

\section{References}

1. McDonald TJ et al (1978) A gastrin releasing peptide from the porcine nonantral gastric tissue. Gut 19:767-774 
2. Walsh J, Dockray GJ, Bunnet et al (1994) Gut peptides. In: Walsh J, Dockray GJ (eds) Gastrin releasing peptide. Raven Press, New York, pp 423-445

3. Modigliani E et al (1990) Immunoreactive gastrin-releasing peptide in medullary thyroid carcinoma. J Clin Endocrinol Metab 71:831-835

4. Thomas SM et al (2005) Gastrin-releasing peptide receptor mediates activation of the epidermal growth factor receptor in lung cancer cells. Neoplasia 7:426-431

5. Gustafson WC et al (2005) Role of gastrointestinal hormones in neuroblastoma. World J Surg 29:281-286

6. Gugger M, Reubi JC (1999) Gastrin-releasing peptide receptors in non-neoplastic and neoplastic human breast. Am J Pathol 155:2067-2076

7. Schwartsmann G et al (2005) MAPK pathway activation in colorectal cancer: a therapeutic opportunity for GRP receptor antagonists. Lancet Oncol 6:444-445

8. Lacoste J, Aprikian AG, Chevalier S (2005) Focal adhesion kinase is required for bombesin-induced prostate cancer cell motility. Mol Cell Endocrinol 235:51-61

9. Schuhmacher J et al (2005) GRP receptor-targeted PET of a rat pancreas carcinoma xenograft in nude mice with a 68 Ga-labeled bombesin(6-14) analog. J Nucl Med 46:691-699

10. Pansky A et al (2000) Gastrin releasing peptide-preferring bombesin receptors mediate growth of human renal cell carcinoma. J Am Soc Nephrol 11:1409-1418

11. Reubi JC et al (2002) Bombesin receptor subtypes in human cancers: detection with the universal radioligand (125)I-[D-TYR(6), beta-ALA(11), PHE(13), NLE(14)] bombesin(6-14). Clin Cancer Res 8:1139-1146

12. Heuser $M$ et al (2005) Expression of gastrin releasing peptide receptor in renal cell carcinomas: a potential function for the regulation of neoangiogenesis and microvascular perfusion. J Urol 173:2154-2159

13. Daniliuc S et al (2003) Hypoxia inactivates inducible nitric oxide synthase in mouse macrophages by disrupting its interaction with alpha-actinin 4. J Immunol 171:3225-3232

14. Leek RD et al (1996) Association of macrophage infiltration with angiogenesis and prognosis in invasive breast carcinoma. Cancer Res 56:4625-4629

15. Siegert A et al (1999) Suppression of the reactive oxygen intermediates production of human macrophages by colorectal adenocarcinoma cell lines. Immunology 98:551-556

16. Condeelis J, Pollard JW (2006) Macrophages: obligate partners for tumor cell migration, invasion, and metastasis. Cell 124:263-266
17. Crowther $\mathrm{M}$ et al (2001) Microenvironmental influence on macrophage regulation of angiogenesis in wounds and malignant tumors. J Leukoc Biol 70:478-490

18. Lewis CE, Pollard JW (2006) Distinct role of macrophages in different tumor microenvironments. Cancer Res 66:605-612

19. De la Fuente M et al (1991) Modulation of phagocytic function in murine peritoneal macrophages by bombesin, gastrin-releasing peptide and neuromedin C. Immunology 73:205-211

20. Del Rio M, De la Fuente M (1994) Chemoattractant capacity of bombesin, gastrin-releasing peptide and neuromedin $\mathrm{C}$ is mediated through PKC activation in murine peritoneal leukocytes. Regul Pept 49:185-193

21. Lemaire I (1991) Bombesin-related peptides modulate interleukin-1 production by alveolar macrophages. Neuropeptides 20:217-223

22. Lemaire I, Jones S, Khan MF (1991) Bombesin-like peptides in alveolar macrophage: increased release in pulmonary inflammation and fibrosis. Neuropeptides 20:63-72

23. Glover $\mathrm{S}$ et al (2005) Transient upregulation of GRP and its receptor critically regulate colon cancer cell motility during remodeling. Am J Physiol Gastrointest Liver Physiol 288:G1274-G1282

24. Matkowskyj KA et al (2003) Expression of GRP and its receptor in well-differentiated colon cancer cells correlates with the presence of focal adhesion kinase phosphorylated at tyrosines 397 and 407. J Histochem Cytochem 51:1041-1048

25. Scott $\mathrm{N}$ et al (2004) Gastrin releasing peptide and gastrin releasing peptide receptor expression in gastrointestinal carcinoid tumours. J Clin Pathol 57:189-192

26. Meloni $\mathrm{F}$ et al (1996) Bombesin enhances monocyte and macrophage activities: possible role in the modulation of local pulmonary defenses in chronic bronchitis. Respiration 63:28-34

27. van Tol EA et al (1991) Intravenous administration of bombesin in man stimulates natural killer cell activity against tumour cells. Neuropeptides 18:15-21

28. Hemmerlein B et al (2000) Expression of acute and late-stage inflammatory antigens, c-fms, CSF-1, and human monocytic serine esterase 1 , in tumor-associated macrophages of renal cell carcinomas. Cancer Immunol Immunother 49:485-492

29. Levine $L$ et al (2003) Bombesin stimulates nuclear factor kappa B activation and expression of proangiogenic factors in prostate cancer cells. Cancer Res 63:3495-3502

30. Busby JE et al (2003) Angiogenesis is not mediated by prostate cancer neuropeptides. Angiogenesis 6:289-293 